\title{
Avaliação do Modelo Global do Geopotencial GOCO05c na Região Sul do Brasil
}

\author{
Evaluation of the Global Geopotential Model GOCO05c in the Southern \\ Region of Brazil
}

Eurico Nicacio ${ }^{1}$

Regiane Dalazoana ${ }^{2}$

Recebido em abril de 2018. Aprovado em novembro de 2018.

\begin{abstract}
RESUMO
O presente trabalho apresenta uma análise quantitativa da adequação do Modelo Global do Geopotencial (MGG) GOCO05C para modelagem de altitude normal-geoidal na região Sul do Brasil. A análise em tela foi inferida de comparações entre valores de altitude normal-geoidal advindos de soluções GNSS/Nivelamento e valores provenientes dos modelos globais combinados EGM2008, EIGEN-6C4 e GOCO05C, segundo as funcionais do geopotencial que melhor representam a altitude normal-geoidal na região altitude geoidal e anomalia de altitude - e em diferentes graus de desenvolvimento, sempre pelo método relativo. Este procedimento foi aplicado sobre as 111 (cento e onze) estações da rede SAT-GPS do IBGE com conexão com a Rede Altimétrica Fundamental Brasileira, as quais são portadoras de informações de altitude elipsoidal e de altitude normalortométrica, ao longo de toda a já mencionada região. Como principal resultado, é possível atestar o desempenho superior do MGG GOCO05C comparativamente com os demais modelos combinados testados quando todos são desenvolvidos até grau 720 , seu grau máximo, e sua teórica igualdade quando todos são utilizados em seus graus máximos individuais. Isto reafirma seu posicionamento como expoente no histórico de desenvolvimento de modelos combinados, com vistas à ampla divulgação de seus resultados e de potencialidade de seu uso.
\end{abstract}

PALAVRAS-CHAVE: Modelo Global do Geopotencial. GOCO05c. Análise quantitativa.

\begin{abstract}
The present paper presents a quantitative analysis of the adequacy of the Global Geopotential Model (GGM) GOCO05C for modeling the normalgeoidal height in the Southern region of Brazil. Such analysis was inferred from comparisons between normal-geoidal height values derived from
\end{abstract}

\footnotetext{
${ }^{1}$ Programa de Pós-graduação em Ciências Geodésicas, Universidade Federal do Paraná, Brasil. E-mail: euriconicaciojr@gmail.com

${ }^{2}$ Programa de Pós-graduação em Ciências Geodésicas, Universidade Federal do Paraná, Brasil. E-mail: regiane@ufpr.br
} 
GNSS/Leveling solutions and values obtained from the combined global models EGM2008, EIGEN-6c4 and GOCO05c, according to the geopotential functionals that best represent the normal-geoidal height at the region geoid height and height anomaly - and in different development degrees, always through the relative method. This procedure was applied to the 111 (one hundred and eleven) stations of IBGE's SAT-GPS network with connection to the Brazilian Altimetric Fundamental Network, which carry information of both ellipsoidal height and normal-orthometric height, throughout the whole already mentioned region. As a main result, it is possible to confirm the superior performance of the GGM GOCO05C compared to the other combined models tested when all are developed up to grade 720 , its maximum degree, and its theoretical equality when all are used at their individual maximum degrees. This reaffirms its position as an exponent in the history of the development of combined models, with a view to the wide dissemination of its results and the potentiality of its use.

KEYWORDS: Global Geopotential Model. GOCO05C. Quantitative analysis.

\section{Introdução}

Conforme Hofmann-Wellenhof e Moritz (2006) e Torge e Müller (2012), o objetivo primordial da Geodésia é determinar a forma e a dimensão da Terra, bem como do campo da gravidade em seu exterior e suas variações temporais. Entretanto, ao longo das últimas décadas este objetivo tem passado por drásticas mudanças, principalmente devido à evolução das técnicas aplicadas em observações e dos métodos computacionais empregados em seu processamento. Concomitantemente a estes avanços, os problemas clássicos da Geodésia foram expandidos, e sua função primária de medição da superfície terrestre foi ampliada para a detecção dos efeitos de mudanças globais e da geodinâmica (DREWES, 2006).

No que diz respeito a importantes marcos da referida evolução, pode-se mencionar a forma diferenciada com que os estudiosos, ao longo dos anos, passaram a observar e analisar dados provenientes do campo da gravidade terrestre. Em espacial, a popularização do sistema GPS (Global Positioning System) nas últimas duas décadas, e mais recentemente do sistema GNSS (Global Navigation Satellite System), revolucionou as atividades que necessitam de posicionamento em função de sua rapidez e precisão na 
obtenção de coordenadas. Este fato impulsionou interesse e necessidade por modelos globais adequados e fidedignos para, por exemplo, proporcionar a determinação de uma superfície equipotencial do campo da gravidade terrestre mais acurada e precisa para aplicações nas áreas de mapeamento e engenharia. Além disso, novas técnicas para obtenção de informações do campo da gravidade a partir de missões satelitais foram desenvolvidas, popularizando o conhecimento e o uso dos Modelos Globais do Geopotencial (MGGs).

Em suma, os MGGs consistem de um conjunto de valores numéricos para certos parâmetros, as estatísticas dos erros a eles associados e uma coleção de expressões matemáticas, valores numéricos e algoritmos particulares, e a providencial aplicação destes dados quando do desenvolvimento do geopotencial em harmônicos esféricos, tal como representado na Equação 1.

$$
\begin{aligned}
W(r, \varphi, \lambda)= & \frac{G M}{r}[1 \\
& +\sum_{n=2}^{\infty} \sum_{m=0}^{n}\left(\frac{a}{r}\right)^{n}\left(C_{n m} \cos (m \lambda)\right. \\
& \left.\left.+S_{n m} \sin (m \lambda)\right) P_{n m}(\sin (\varphi))\right]+\frac{1}{3} \omega^{2} r^{2}\left[1-P_{20}(\sin (\varphi))\right]
\end{aligned}
$$

Nesta equação, $a$ é o semieixo maior do elipsoide associado ao modelo, $\omega$ é a velocidade angular de rotação da Terra, $C_{n m}$ e $S_{n m}$ são os coeficientes do desenvolvimento em séries, $G M$ é a constante gravitacional geocêntrica associada ao modelo, $(r, \varphi, \lambda)$ são as coordenadas geocêntricas do ponto de cálculo, a saber: $r$ é a distância ao geocentro do modelo adotado, $\varphi$ é a latitude geodésica e $\lambda$ é a longitude geodésica, e $P_{n m}$ representa as funções de Legendre associadas com grau $n$ e ordem $m$.

Ainda segundo a referência citada, um MGG deve ser capaz de suportar tais cálculos em quaisquer pontos arbitrários, localizados sobre ou acima da superfície terrestre, de maneira precisa e eficiente, caracterizando sua 
globalidade. Estes aspectos, aliados à consistência interna de informações e à modelagem física precisa, propiciam exatidão e confiabilidade aos resultados fornecidos.

Entretanto, inseridos neste contexto, diferentes MGGs foram desenvolvidos ao longo das últimas cinco décadas, cada um com fontes e conjuntos de dados atinentes ao propósito a que se destina. Na atualidade, em virtude da complexidade de obtenção e manutenção destes modelos, bem como da necessidade de controle e normatização de todas as variáveis relacionadas, tendo em mente o benefício de usuários ao redor do planeta, sua gestão é realizada globalmente pelo ICGEM - International Centre for Global Earth Models, Centro Internacional para Modelos Globais Terrestres. Este é um dos cinco serviços coordenados pelo IGFS - International Gravity Field Service, Serviço Internacional do Campo da Gravidade - subordinados à IAG International Association of Geodesy, Associação Internacional de Geodésia (BARTHELMES e KÖHLER, 2016). Sua base de dados conta com 168 (cento e sessenta e oito) modelos estáticos, dos quais se destacam os MGGs combinados, que conjugam informações provenientes de observações de órbitas satelitais, gravimetria terrestre e altimetria (quer seja proveniente de modelos digitais de elevação, em continentes e regiões costeiras, quer seja proveniente de altimetria por satélites, nos oceanos) para solução da modelagem do geopotencial (SEEBER, 2003).

\section{$2 \quad$ Material e Métodos}

2.1 Área de estudo

Para realizar a avaliação quantitativa que ora se propõe, elencou-se como área de estudo a região Sul do Brasil, principalmente em virtude de a mesma conter o Datum Vertical Brasileiro, em Imbituba - Santa Catarina (DVB-I) e da consequente necessidade de se conhecer o comportamento do principais MGGs da atualidade em seu entorno. Destarte, foram selecionadas

Rev. Bras. de Cartografia, vol. 70, Edição Especial "XXVII Congresso Brasileiro de 
as 111 (cento e onze) estações da rede SAT-GPS do IBGE com conexão com a Rede Altimétrica Fundamental Brasileira (RAFB), doravante denominadas estações GNSS/RN.

Estas estações foram selecionadas pois são portadoras das duas informações necessárias para obtenção de modelagem normal-geoidal com o uso de GNSS e nivelamento, a saber: altitude elipsoidal e altitude normalortométrica. Note-se que a análise em tela foi desenvolvida anteriormente ao reajustamento da RAFB em termos de números geopotenciais, o qual culminou com representação de suas referências de nível por meio de altitudes normais (IBGE, 2018).

Neste contexto, a Figura 1 exibe a extensão da área de estudo, sendo as estações GNSS/RN simbolizadas pelos pontos vermelhos.

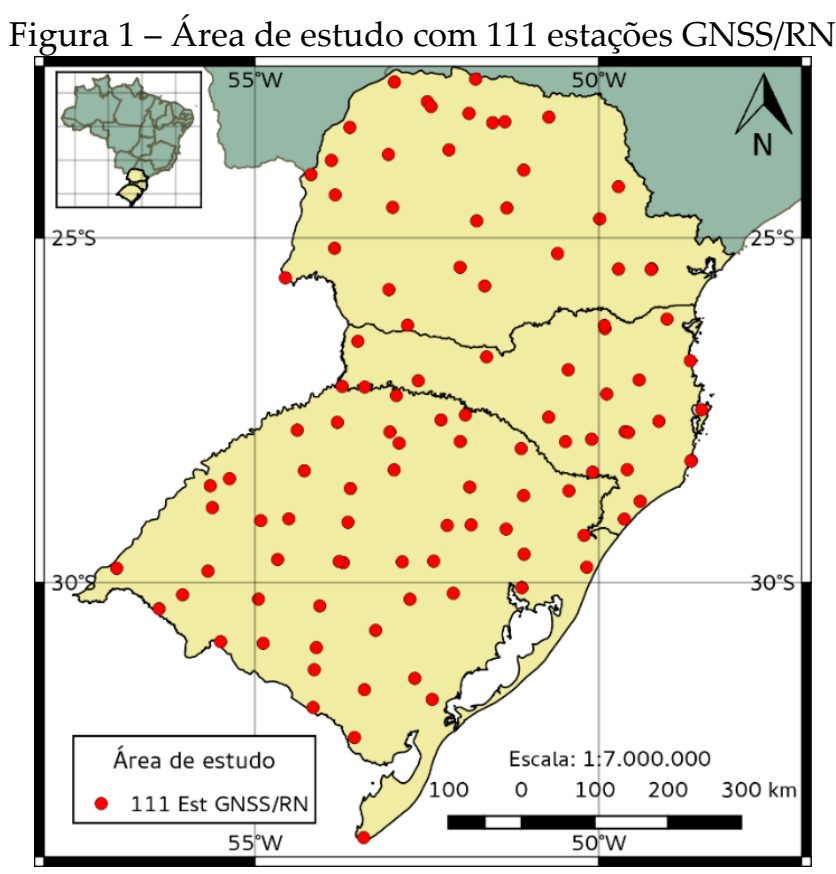

Fonte: Elaborada pelos autores.

\subsection{Modelos Globais do Geopotencial empregados}

Conforme apresentado na Introdução, optou-se pelo uso dos MGGs combinados, que conjugam informações provenientes de diferentes fontes para solução da modelagem do geopotencial. Neste contexto, tal como descrito 
por Pavlis et al. (2012), o primeiro modelo baseado não apenas na solução de estimativas de um conjunto de anomalias de gravidade provenientes de observações de órbitas satelitais, mas também providos de observações gravimétricas, observações sobre a topografia do terreno e de altimetria por satélite foi o EGM2008.

Entretanto, desde seu lançamento, diferentes técnicas e métodos de processamento tem sido desenvolvidas e aplicadas em benefício de uma melhor compreensão e extração de informações sobre o campo da gravidade das fontes cabíveis. Para fins de brevidade, o presente trabalho omite a análise conceitual sobre a evolução dos MGGs combinados ao longo da última década, podendo esta ser consultada em Nicacio e Dalazoana (2017).

A partir da análise indicada por estes autores, foram selecionados para a presente avaliação os modelos globais do geopotencial EGM2008, EIGEN6c4 e GOCO05c. A obtenção de seus extratos foi realizada de maneira pontoa-ponto, segundo cada uma das 111 estações GNSS/RN, para que fosse possível preservar as características e a precisão de cada modelo.

Utilizou-se, para este fim, a aplicação standalone SPGG v2.0 (NICACIO, 2016), a qual obtém extratos pontuais de MGGs segundo funcionais e características definidoras especificadas pelo usuário em uma interação direta com o serviço web do ICGEM. Uma outra possibilidade de obtenção, em forma de grid no entorno da área e com posterior interpolação em pontos de interesse, não preservaria estas características, tal como indicado por Nicacio e Dalazoana (2016). Neste sentido, a Tabela 1 apresenta a listagem de modelos e graus de desenvolvimento dos extratos empregados no presente trabalho.

Tabela 1- MGGs e graus de desenvolvimento empregados no trabalho.

\begin{tabular}{c|c|c|c}
\hline MGG & \multicolumn{3}{|c}{ Graus de desenlvovimento } \\
\hline EGM2008 & 2190 & 720 & 360 \\
\hline EIGEN-6c4 & 2190 & 720 & 360 \\
\hline GOCO05c & - & 720 & 360 \\
\hline
\end{tabular}

Fonte: elaborada pelos autores. 


\subsection{Modelagem da Altitude Normal-Geoidal}

A modelagem da altitude normal-geoidal por meio de soluções GNSS/nivelamento abordada neste trabalho leva em consideração o fato de que, no Brasil, as altitudes apresentadas na RAFB à época de realização dos trabalhos eram altitudes normais-ortométricas, $H^{\text {Nort }}$, ou seja, são oriundas de altitudes niveladas às quais aplica-se uma correção normal-ortométrica para fins de correção do não-paralelismo das superfícies equipotenciais do campo da gravidade (LUZ, 2008).

Neste sentido, Ferreira et al. (2013) discorrem que o fato do comportamento das altitudes normais-ortométricas não apresentarem um significado completamente físico faz com que estas estejam referidas a uma superfície de referência clássica, como o geoide ou o quase-geoide. Desta forma, o tradicional equacionamento que relaciona altitudes elipsoidais $\mathrm{h}$ e altitudes genuinamente ortométricas $H^{\text {ort }}$ por meio da altitude geoidal $N, h=$ $H^{\text {ort }}+N$, não pode ser utilizado livremente como se as altitudes da RAFB fossem, de fato, altitudes ortométricas.

Em virtude disso, Ferreira et al. (2013) ainda descreve a dependência entre as altitudes elipsoidais $h$ e as altitudes normais-ortométricas $H^{N o r t}$ aos moldes da Equação 2, na qual $\eta$ é por eles denominada como altitude normalgeoidal, em analogia à altitude normal-ortométrica, nomenclatura esta mantida neste trabalho.

$$
h=H^{N O r t}+\eta
$$

A altitude normal-geoidal pode, ainda, ser melhor modelada pela própria altitude geoidal $N$ ou pela anomalia de altitude $\zeta$, dada pelo afastamento entre a superfície terrestre e o teluroide, medido ao longo da normal ao ponto de estudo (JEKELI, 2000), dependendo da área que se estuda. Ferreira et al. (2011), por exemplo, verificaram que, tomadas diferentes aproximações para a separação entre o geoide e o quase-geoide, a 
altitude normal-geoidal é levemente melhor modelada pela anomalia de altitude do que pela altitude geoidal, em um estudo de caso aplicado a estações da região sul do Brasil.

Entretanto, para eliminar erros aditivos inerentes ao sistema de processamento e ao modo de obtenção dos MGGs, Featherstone (2001), Arana (2000) e Nicacio (2017) propõem a utilização do método relativo em substituição ao método absoluto, o qual se baseia, no contexto deste trabalho, na utilização de um ponto origem $P_{0}$ como referência, com altitude normalortométrica $H_{0}^{\text {Nort }}$, altitude elipsoidal $h_{0}$ e altitude normal-geoidal $\eta_{0}$ conhecidas e pela abordagem matemática apresentada na Equação 3.

$$
\begin{gathered}
\Delta h=\Delta H^{N O r t}+\Delta \eta \rightarrow \\
\eta_{P}=\eta_{0}+h_{P}-h_{0}-H_{P}^{N O r t}+H_{0}^{N O r t}
\end{gathered}
$$

Conforme reforçado por SIRGAS (2016), esta é a alternativa mais aconselhável na atualidade, principalmente sob o aspecto de consecução de sistemas de referência vertical. Segundo esta referência, as atuais técnicas geodésicas, principalmente aquelas apoiadas por posicionamento e navegação satelital, apresentam requisitos de precisão superiores àqueles propiciados pela equação absoluta tradicional. Sendo assim, a utilização do método relativo para correlacionar altitudes elipsoidal e normal-ortométrica com a altitude normal-geoidal, foi adotada no presente trabalho.

Frisa-se, ainda, que pra fins de manipulação das variáveis envolvidas, foi necessário realizar a compatibilização do sistema de maré permanente, consonante ao preconizado por Ekman (1989), Mäkinen e Ihde (2009) e Tenzer et al. (2011). Desta forma, todas as variáveis foram compatibilizadas para o sistema tide-free, ou livre de maré, utilizando formulação matemática constante da última referência. 


\subsection{Critério de avaliação empregado}

O critério utilizado para escolha, em um dado ponto de cálculo $P$, de qual modelo melhor se adequa para realizar a determinação altimétrica levou em consideração a metodologia apresentada na Equação 4:

$$
\left\{\begin{array}{l}
{\left[\eta_{P}\right]_{E G M 2008}} \\
{\left[\eta_{P}\right]_{E I G E N 6 c 4}} \\
{\left[\eta_{P}\right]_{G O C O 05 c}}
\end{array} \Rightarrow \text { mín } \theta_{P}=\left|\eta_{P}^{\text {ref }}-\eta_{P}^{\text {calc }}\right|\right.
$$

Na citada equação, deve ocorrer a minimização de um fator $\theta_{P}$, igual ao módulo da diferença entre a altitude normal-geoidal de referência $\eta_{P}^{\text {ref }}$, obtida por meio da Equação 3, e a altitude normal-geoidal calculada $\eta_{P}^{\text {calc }}$, obtida diretamente como funcional do modelo em questão.

No que diz respeito ao padrão adotado para o ponto zero $P_{0}$, sua escolha foi determinada em função daquele que, para todo o conjunto de pontos, foi capaz de minimizar o fator $\theta_{m}$, tal como regrado pela Equação 5.

$$
P_{0} \Rightarrow \min \theta_{m}=\frac{1}{n} \sum_{i=1}^{n} \theta_{i}
$$

A modelagem e a análise em diferentes graus de desenvolvimento e das diferentes funcionais do geopotencial para representar a altitude normalgeoidal foi realizada com formulação similar à Equação 4. Esta encontra-se indiretamente representada nos resultados cabíveis, sendo seus pormenores omitidos do presente trabalho.

\section{Resultados e Discussões}

Uma descrição com os principais resultados da avaliação ora desenvolvida é apresentada na Tabela 2, a qual contém os valores de 
diferenças mínima, média e máxima para cada configuração - leia-se, para cada especificação de MGG, funcional e grau de desenvolvimento juntamente com seu erro médio quadrático (EMQ), para todo o conjunto de pontos. A partir das informações nela contidas, pode-se observar os principais resultados apresentados ao longo deste item.

Tabela 2 - Resumo dos principais resultados obtidos na avaliação do MGG GOCO05c

Graus de desenvolvimento e Funcionais do Geopotencial

\begin{tabular}{c|c|c|c|c|c|c|c}
\hline \multirow{4}{*}{ MGG } & \multirow{2}{*}{ Dif (m) } & \multicolumn{2}{|c|}{$\mathbf{2 1 9 0}$} & \multicolumn{2}{c|}{$\mathbf{7 2 0}$} & \multicolumn{2}{c}{$\mathbf{3 6 0}$} \\
\cline { 3 - 8 } & & $\begin{array}{c}\text { Glt } \\
\text { Geoid }\end{array}$ & $\begin{array}{c}\text { Anom } \\
\text { Alt }\end{array}$ & $\begin{array}{c}\text { Alt } \\
\text { Geoid }\end{array}$ & $\begin{array}{c}\text { Anom } \\
\text { Alt }\end{array}$ & $\begin{array}{c}\text { Alt } \\
\text { Geoid }\end{array}$ & $\begin{array}{c}\text { Anom } \\
\text { Alt }\end{array}$ \\
\hline \multirow{4}{*}{ EGM2008 } & MÍN & 0,0013 & 0,0006 & 0,0004 & 0,0005 & 0,0037 & 0,0007 \\
\cline { 2 - 8 } & MÉD & $\mathbf{0 , 1 1 3 9}$ & $\mathbf{0 , 1 0 2 7}$ & $\mathbf{0 , 1 2 0 3}$ & $\mathbf{0 , 1 0 9 2}$ & $\mathbf{0 , 1 5 9 8}$ & $\mathbf{0 , 1 5 0 4}$ \\
\cline { 2 - 8 } & MÁX & 0,6440 & 0,6429 & 0,6497 & 0,6585 & 0,8452 & 0,8525 \\
\cline { 2 - 8 } & EMQ & 0,1037 & 0,1023 & 0,1083 & 0,1060 & 0,1334 & 0,1313 \\
\hline \multirow{4}{*}{ EIGEN-6C4 4} & MÍN & 0,0013 & 0,0013 & 0,0009 & 0,0017 & 0,0009 & 0,0000 \\
\cline { 2 - 8 } & MÉD & $\mathbf{0 , 1 0 7 4}$ & $\mathbf{0 , 0 9 7 2}$ & $\mathbf{0 , 1 1 7 9}$ & $\mathbf{0 , 1 0 6 0}$ & $\mathbf{0 1 4 7 8}$ & $\mathbf{0 , 1 3 7 6}$ \\
\cline { 2 - 8 } & MÁX & 0,6115 & 0,6140 & 0,6299 & 0,6228 & 0,711 & 0,8012 \\
\cline { 2 - 8 } & EMQ & 0,0950 & 0,0935 & 0,0952 & 0,0940 & 0,1348 & 0,1294 \\
\hline \multirow{4}{*}{ GOCO05C } & MÍN & - & - & 0,0008 & 0,0003 & 0,0020 & 0,0004 \\
\cline { 2 - 8 } & MÉD & - & - & $\mathbf{0 , 1 1 0 2}$ & $\mathbf{0 , 0 9 9 3}$ & $\mathbf{0 , 1 4 8 2}$ & $\mathbf{0 , 1 3 9 8}$ \\
\cline { 2 - 8 } & MÁX & - & - & 0,6346 & 0,6232 & 0,7888 & 0,8002 \\
\cline { 2 - 8 } & EMQ & - & - & 0,1044 & 0,1010 & 0,1344 & 0,1296 \\
\hline
\end{tabular}

Fonte: Elaborada pelos autores.

Primeiramente, deve-se observar que o desempenho da funcional anomalia de altitude foi superior à funcional altitude geoidal para modelagem da altitude normal-geoidal na área de estudo. Este resultado já era esperado, pois está em sincronia com resultados anteriores obtidos por Ferreira et al. (2013) também na região Sul do Brasil.

$\mathrm{Na}$ sequência, em uma primeira análise, observa-se que, utilizando extratos dos modelos desenvolvidos até grau 720, grau máximo possível para o modelo ora avaliado, a discrepância média entre os valores comparados para GOCO05c, EIGEN-6c4 e EGM2008 é de 0,1102 $m=11,02 \mathrm{~cm}, 0,1179 \mathrm{~m}=$ $11,79 \mathrm{~cm}$ e $0,1203 \mathrm{~m}=12,03 \mathrm{~cm}$ para a funcional altitude geoidal, e $0,0993 \mathrm{~m}=9,93 \mathrm{~cm}, 0,1060 \mathrm{~m}=10,60 \mathrm{~cm}$ e $0,1092 \mathrm{~m}=10,92 \mathrm{~cm}$ para a 
funcional anomalia de altitude, respectivamente, comprovando seu melhor desempenho quando todos os modelos são utilizados até este grau de desenvolvimento. Entretanto, em uma segunda análise, utilizando-se todos os MGGs em seus graus máximos individuais possíveis - grau 720 para GOCO05c e grau 2190 para EGM2008 e EIGEN-6c4 - a discrepância média entre os fatores comparados para GOCO05c, EIGEN-6c4 e EGM2008 é de $0,1101 \mathrm{~m}=11,01 \mathrm{~cm}, 0,1074 \mathrm{~m}=10,74 \mathrm{~cm}$ e $0,1139 \mathrm{~m}=11,39 \mathrm{~cm}$ para a funcional altitude geoidal, e $0,0993 \mathrm{~m}=9,93 \mathrm{~cm}, 0,0971 \mathrm{~m}=9,71 \mathrm{~cm}$ e $0,1027 \mathrm{~m}=10,27 \mathrm{~cm}$ para a funcional anomalia de altitude, respectivamente.

Este resultado coloca o MGG GOCO05c em vantagem em relação ao MGG EGM2008, mas em desvantagem milimétrica para o MGG EIGEN-6c4, sendo esta quantificada em 2,7 $\mathrm{mm}$ para altitude geoidal e 2,2 $\mathrm{mm}$ para anomalia de altitude. Justifica-se esta desvantagem principalmente pelo erro de comissão entre os graus 720 e 2190; contudo, considerando-se a precisão típica centimétrica das informações altimétricas na área de estudo, a diferença encontrada não é significativa, existindo uma equivalência estatística dos resultados auferidos e sendo o desempenho dos modelos utilizados compatível, mesmo com a grande diferença entre graus máximos de desenvolvimento.

\section{Conclusão}

Através do estudo ora desenvolvido, é possível atestar o desempenho superior do MGG GOCO05c comparativamente com os demais modelos combinados testados (EGM2008 e EIGEN-6c4) quando todos são desenvolvidos até grau 720, seu grau máximo, e sua equivalência estatística quando todos são utilizados em seus graus máximos individuais. Sua desvantagem milimétrica em relação ao último é plenamente justificável por erros de omissão devido à discrepância entre graus de desenvolvimento de ambos. 
Este resultado reafirma, portanto, o posicionamento do GOCO05c como expoente no histórico de desenvolvimento de modelos combinados (Nicacio e Dalazoana, 2017), com vistas à ampla divulgação de seus resultados e de potencialidade de seu uso.

\section{Referências}

ARANA, J. M. O uso do GPS na elaboração da carta geoidal. Tese de doutoramento. Universidade Federal do Paraná, Programa Pós-Graduação em Ciências Geodésicas, Curitiba, 2001. 181p.

BARTHELMES, F.; KÖHLER, W. International Centre for Global Earth Models (ICGEM). Journal of Geodesy, vol. 90, nº 10, 2016. pp. 907-1205.

DREWES, H. The changing objectives in geodetic research. Zeitschrift für Geodäsie, Geo-Information und Landmanagement, Vol. 131, N 5, 2006. pp. 1-7.

EKMAN, M. Impacts of Geodynamic Phenomena on Systems for Height and Gravity. Bulletin Géodésique, vol. 63, nº 3, 1989. pp. 281-296.

FEATHERSTONE, W. E. Absolute and relative testing of gravimetric geoid models using Global Positioning System and orthometric height data. Computers \& Geosciences, vol. 27, nº 7, 2001. pp. 807-814.

FERREIRA, V. G. et al. A separação entre o geoide e o quase-geiode: uma análise no contexto brasileiro. Revista Brasileira de Cartografia, v. 63, 2011. FERREIRA, V. G. et al. Validation of GOCE gravity field models using GPSleveling data and EGM08: a case study in Brazil. Journal of Geodetic Science, vol. 3, $n^{\circ}$ 3, 2013. pp. 209-218.

HOFMANN-WELlenhoF, B.; MORITZ, H. Physical Geodesy. $2^{\mathrm{a}}$ ed. Austria: Spring-Verlag Wien, 2006. 403p.

IBGE. Reajustamento da Rede Altimétrica com Números Geopotenciais 2018. Relatório Técnico, Instituto Brasileiro de Geografia e Estatística, Curitiba, 2018. 55p.

JEKELI, C. Heights, the Geopotential, and Vertical Datums. Report n. 459. Geodetic Science and Surveying, Department of Civil and Environmental 
Engineering and Geodetic Science. The Ohio State University - Columbus, Ohio, 2000.

LUZ, R. T. Estratégias para modernização da componente vertical do Sistema Geodésico Brasileiro e sua integração ao SIRGAS. Tese de doutoramento. Universidade Federal do Paraná, Programa Pós-Graduação em Ciências Geodésicas, Curitiba, 2008. 232p.

MÄKINEN, J.; IHDE, J. The permanent tide in height systems. In: Sideris M. G. (eds) Observing our Changing Earth. International Association of Geodesy Symposia, vol. 133, Berlin, 2009. pp 81-88.

NICACIO, E. Single Point GEM Generator. Disponível em: $<$ http://euriconicacio.github.io/spgg>. 2016.

NICACIO, E. Alternativa para determinação de altitudes normaisortométricas na Amazônia Legal Brasileira. Dissertação de mestrado. Universidade Federal do Paraná, Programa Pós-Graduação em Ciências Geodésicas, Curitiba, 2017. 229p.

NICACIO, E.; DALAZOANA, R. Análisis de extractos de Modelos Globales de Geopotencial generados punto-a-punto a partir de una aplicación standalone, Anais do Simposio SIRGAS 2016, Quito, Equador, 2016.

NICACIO, E.; DALAZOANA, R. Passado e presente dos Modelos Globais do Geopotencial: uma abordagem conceitual sobre sua evolução. Revista Eletrônica Multidisciplinar FACEAR, vol. 2, nº 6, 2017. pp. 1-15.

PAVLIS, N. K. et al. The development and evaluation of the Earth Gravitational Model 2008 (EGM2008). Journal of Geophysical Research, vol. 117, N B04406, 2012. pp. 1-38.

SIRGAS. Report on the SIRGAS Working Group III "Vertical Datum" Workshop. Relatório Técnico, Instituto Brasileiro de Geografia e Estatística, Quito, Equador, 2016.2p.

SEEBER, G. Satellite Geodesy: foundations, methods and applications. Berlin-New York: Walter de Gruyter, 2003. 612p.

TENZER, R. et al. Assessment of the LVD offsets for the normal-orthometric heights and different permanent tide systems - a case study of New Zealand. Applied Geomatics, vol. 3, n 1,2011 . pp. 1-8. 
TORGE, W.; MÜLLER, J. Physical Geodesy. Berlin-Boston: Walter de Gruyter, 2012. 445p.

Rev. Bras. de Cartografia, vol. 70, Edição Especial "XXVII Congresso Brasileiro de Cartografia”, 2018. pp. 1537 - 1550. 